\title{
Hypoxia induces immunogenic cell death of cancer cells by enhancing the exposure of cell surface calreticulin in an endoplasmic reticulum stress-dependent manner
}

\author{
YU KYEONG HAN ${ }^{1}$, GA-YOUNG PARK ${ }^{1,2}$, MIN JI BAE ${ }^{1}$, JOONG SUN KIM ${ }^{3}$, \\ WOL SOON JO ${ }^{1}$ and CHANG GEUN LEE ${ }^{1}$
}

\begin{abstract}
${ }^{1}$ Department of Radiation Biology, Research Center, Dongnam Institute of Radiological and Medical Sciences, Busan 46033;
${ }^{2}$ Department of Molecular Biology, College of Natural Sciences, Pusan National University, Busan 46241;

${ }^{3}$ K-Herbal Medicinal Research Center, Korea Institute of Oriental Medicine, Daejeon 34054, Republic of Korea
\end{abstract}

Received March 6, 2019; Accepted September 6, 2019

DOI: 10.3892/ol.2019.10986

\begin{abstract}
Hypoxia is associated with resistance to anticancer therapies. Additionally, it is involved in the immune evasion of cancer cells by inducing an immunosuppressive microenvironment. However, the role of hypoxia in modulating the immunogenicity of cancer cells remains unknown. Hypoxia is known to induce endoplasmic reticulum (ER) stress, which serves a key role in inducing the cell surface exposure of calreticulin, a marker of immunogenic cell death. The present study investigated whether hypoxia influenced the immunogenicity of cancer cells using FACS, western blot analysis and syngenic mouse tumor model. The results revealed that hypoxia induced the cell surface exposure of calreticulin in human and mouse breast cancer cell lines depending on ER stress. Enhanced cell surface exposure of calreticulin induced by hypoxia resulted in an increase in anticancer immunity in a mouse model, which suggested that hypoxia induced immunogenic cell death. Notably, hypoxia did not significantly modulate the cell surface exposure of CD47, an antagonist of calreticulin function in cancer immunogenicity. These results suggest that hypoxia may enhance the immunogenicity of cancer cells themselves, in addition to its role in inducing an immunosuppressive cancer microenvironment.
\end{abstract}

Correspondence to: Dr Chang Geun Lee or Dr Wol Soon Jo, Department of Radiation Biology, Research Center, Dongnam Institute of Radiological and Medical Sciences, 40 Jwadong-gil, Jangan-eup, Gijang-gun, Busan 46033, Republic of Korea

E-mail: cglee@dirams.re.kr

E-mail: sailorjo@dirams.re.kr

Key words: immunogenic cell death, calreticulin, hypoxia, endoplasmic reticulum stress, CD47

\section{Introduction}

Hypoxia, which commonly occurs in the majority of solid tumors, is associated with resistance to anticancer therapies, including chemotherapy and radiotherapy $(1,2)$. Cancer cells become resistant to anticancer therapies during hypoxia due to their adaptation to lower oxygen levels, which alters the gene expression of metabolic enzymes partially mediated by hypoxia-inducible factors $1 \alpha$ and $2 \alpha$ (3). In addition to transcriptional regulation, hypoxia strongly suppresses the translation rate by inhibiting the expression of eukaryotic initiation factors 2 and $4 \mathrm{~F}$ (4). The endoplasmic reticulum (ER) stress and mTOR signaling pathways serve key roles in the hypoxia-mediated inhibition of eukaryotic initiation factors (4).

Despite the well-known role of hypoxia in the resistance to anticancer therapies, its roles in cancer immunity, to the best of our knowledge, have not been extensively studied. Recent studies have demonstrated that hypoxia promotes cancer progression by impairing anticancer immunity via various mechanisms (5-8). Cancer cells in hypoxic regions release molecules that induce the differentiation of tumor-associated macrophages (TAM) into immunosuppressive phenotypes, including M2-type TAMs, or recruit myeloid-derived suppressor cells $(5,6)$. ER stress in cancer cells, which can also be induced by hypoxia, and low nutrient supply can be transmitted to dendritic cells and impair their ability to prime CD8 ${ }^{+}$ $\mathrm{T}$ cells $(7,8)$. These findings suggest that hypoxia is generally associated with the suppression of anticancer immunity.

Different anticancer therapies result in different types of cancer cell death in terms of immunogenicity. Cancer cell death caused by doxorubicin or irradiation is strongly immunogenic, whereas cancer cell death caused by cisplatin is poorly immunogenic (9). The cell surface exposure of calreticulin has been identified as a key feature in determining immunogenic cell death (9). Activation of the ER stress signaling pathway is involved in the cell surface exposure of calreticulin (10).

Hypoxia induces ER stress in cancer cells. The ER stress signaling pathway is one of the main mechanisms underlying the increased cell surface exposure of calreticulin, a marker of immunogenic cell death $(9,10)$. The present study 
investigated the role of ER stress induced by hypoxia in the immunogenicity of cancer cells. It was identified that hypoxia increased the cell surface exposure of calreticulin in an ER stress-dependent manner, resulting in enhanced immunogenicity of cancer cells. Compared with the efficient increase in the exposure of cell surface calreticulin induced by hypoxia, the cell surface exposure of CD47, an anti-phagocytic signal that antagonizes the activity of calreticulin in phagocytosis, was not efficiently induced by hypoxia. Therefore, hypoxia may enhance the immunogenicity of cancer cells in addition to inducing an immunosuppressive cancer microenvironment. These results may be useful in understanding the role of hypoxia in cancer immunity to design effective anticancer immunotherapies.

\section{Materials and methods}

Cell culture. MCF7 and MDA-MB-231 cells were purchased from the American Type Culture Collection. Both cells were cultured in Dulbecco's Modified Eagle medium (DMEM) supplemented with 10\% FBS, 2 mM L-glutamine, $100 \mathrm{U} / \mathrm{ml}$ penicillin, $100 \mu \mathrm{g} / \mathrm{ml}$ streptomycin at $37^{\circ} \mathrm{C}$ in a $5 \% \mathrm{CO}_{2}$ atmosphere and irradiation were performed as described previously $(11,12)$. The 4 TO7 cells were established by Dr Fred R. Miller at Wayne State University and were cultured in DMEM supplemented with $10 \% \mathrm{FBS}$, $2 \mathrm{mM}$ L-glutamine, $100 \mathrm{U} / \mathrm{ml}$ penicillin, $100 \mu \mathrm{g} / \mathrm{ml}$ streptomycin at $37^{\circ} \mathrm{C}$ in a $5 \% \mathrm{CO}_{2}$ atmosphere (13). A total of $5 \times 10^{6}$ cells were seeded in $150 \mathrm{~mm}$ dishes and following $36 \mathrm{~h}$, cells of $\sim 70 \%$ confluence were exposed to $2 \mu \mathrm{M}$ doxorubicin (Sigma-Aldrich; Merck KGaA) or $300 \mu \mathrm{M}$ cisplatin (Sigma-Aldrich; Merck KGaA) at $37^{\circ} \mathrm{C}$ for $24 \mathrm{~h}$. A total of $5 \times 10^{6}$ cells were seeded in $150 \mathrm{~mm}$ dishes and following $36 \mathrm{~h}$, cells of $\sim 70 \%$ confluence were irradiated with $10 \mathrm{~Gy}$ at room temperature and harvested via fluorescence-activated cell sorting (FACS) analysis $24 \mathrm{~h}$ later. Cells were exposed to hypoxia at $37^{\circ} \mathrm{C}$ for $48 \mathrm{~h}$ in an anaerobic system (Thermo Fisher Scientific, Inc.) using mixed gases $\left(1 \% \mathrm{O}_{2}, 5 \% \mathrm{CO}_{2}\right.$ and $\mathrm{N}_{2}$ balance). The oxygen concentration was monitored using an $\mathrm{O}_{2}$ sensor (New Cosmos Electric Co., Ltd.) prior to hypoxia treatment. A total of $5 \times 10^{6}$ cells were seeded in $150 \mathrm{~mm}$ dishes and following $36 \mathrm{~h}$, cells of $\sim 70 \%$ confluence were treated with $1 \mathrm{mM}$ tauroursodeoxycholic acid (TUDCA; Sigma-Aldrich; Merck KGaA) or 5 mM 4-phenylbutyrate (4-PBA; Sigma-Aldrich; Merck KGaA) at $37^{\circ} \mathrm{C}$ for $30 \mathrm{~min}$ prior to hypoxia treatment.

Flow cytometry analysis. For the analysis of cell surface exposure of calreticulin and CD47, the cells were harvested, washed with PBS and stained with anti-calreticulin antibody (cat. no. 12238; 1:100; Cell Signaling Technology, Inc.), fluorescein-labeled anti-rabbit immunoglobulin $\mathrm{G}(\mathrm{IgG}$; cat. no. 554020;1 $1 \mu \mathrm{g} / \mathrm{ml}$; BD Biosciences) or AlexaFluor ${ }^{\circledR}$ 647-anti-CD47 antibodies (cat. no. 563584; $1 \mu \mathrm{g} / \mathrm{ml}$; BD Biosciences). For determination of the total cell expression of calreticulin, the cells were harvested, permeabilized with Cytofix/Cytoperm (BD Biosciences), and stained with anti-calreticulin antibody and fluorescein-labeled anti-rabbit IgG. The cells were analyzed on a FACS Aria (Becton, Dickinson and Company) using FACSDiva software v6.1.3
(BD Biosciences) to evaluate cell surface exposure and total cell expression.

Western blot analysis. Protein samples were prepared using extraction buffer [50 mM Tris- $\mathrm{HCl}, \mathrm{pH} 7.4,250 \mathrm{mM} \mathrm{NaCl}$, $5 \mathrm{mM}$ EDTA, 0.5\% NP40, $10 \mathrm{mM} \mathrm{NaF}, 1 \mathrm{mM} \mathrm{Na} \mathrm{VO}_{4}$, $1 \mathrm{mM}$ DTT, $1 \mathrm{mM}$ PMSF and $1 \mathrm{X}$ Protease Inhibitor Cocktail (cat. no. 11697498001; Merck KGaA)] and concentrations of protein samples were measured using Bio-Rad Protein assays (cat. no. 5000006; Bio-Rad Laboratories, Inc.). Protein samples $(10 \mu \mathrm{g})$ were separated on a $4-15 \%$ gradient SDS-PAGE gel (cat. no. 456-1084; Bio-Rad Laboratories, Inc.) and transferred to a PVDF membrane (cat. no. 03-010-040-001; Roche Diagnostics GmbH). The membrane was blocked with $10 \%$ skimmed milk (cat. no. 90002-594; Difco; BD Biosciences) in PBST (PBS with $0.1 \%$ Tween) at room temperature (RT) for $30 \mathrm{~min}$ and probed with anti-calreticulin (cat. no. 12238; 1:1,000 in 10\% skimmed milk in PBST; Cell Signaling Technology, Inc.) and anti-actin (cat. no. A2228; 1:3,000 in 10\% skip milk in PBST; Sigma-Aldrich; Merck KGaA) antibodies at RT for $2 \mathrm{~h}$. This was followed by probing with HRP-conjugated anti-rabbit (cat. no. sc-2004; 1:1,000 in 10\% skip milk in PBST; Santa Cruz Biotechnology, Inc.) and HRP-conjugated anti-mouse (cat. no. sc-2005; 1:1,000 in 10\% skip milk in PBST; Santa Cruz Biotechnology, Inc.) secondary antibodies at RT for $1 \mathrm{~h}$. The membrane was incubated with chemiluminescent reagent (ECL Select Western Blottng Detection Reagent; cat. no. RPN2235; GE Healthcare) and then subjected to analysis with a Fusion Fx5 image analyzer (Fusion-CAPT software, Vilber Lourmat).

Animal experiments. Animal studies were conducted under specific pathogen-free conditions (temperature, $22 \pm 3^{\circ} \mathrm{C}$; humidity: 50 $\pm 20 \mathrm{RH}$; illumination, 150-300 Lux; light/dark cycle, 8 am- 8 pm; access to food and water, available anytime) and approved by the Ethics Committee on the Use and Care of Animals of the Dongnam Institute of Radiological and Medical Sciences (Busan, Republic of Korea; approval no. DIRAMS AEC-2015-008). Female Balb/c mice $n=120$ ) were purchased from Japan SLC, Inc. and were used for each experiment (age, 6 weeks; weight, 15-20 g; n=12 per group). Following various treatments (10 Gy irradiation, doxorubicin, cisplatin and culture under hypoxic conditions), $1 \times 10^{6} 4 \mathrm{TO} 7$ cells were subcutaneously injected into the left thighs. At 7 days post-injection, $5 \times 10^{5}$ live $4 \mathrm{TO} 7$ cells were injected into the right thighs. Tumor volume was calculated using the equation: Volume=width ${ }^{2} \times$ length $x 0.52$. Mice with tumor sizes $<150 \mathrm{~mm}^{3}$ were considered tumor-free.

Statistical analysis. Each experiment was repeated at least twice. Results are expressed as the mean \pm SD. To determine statistical significance, the data were analyzed using SPSS statistical software for Windows (version 18.0; SPSS, Inc.). Student's t-test (unpaired) was performed to determine significant differences between two groups, and one-way analysis of variance with Dunnett's post hoc test for multiple comparisons was performed to determine significant differences among more than two groups. $\mathrm{P}<0.05$ was considered to indicate a statistically significant difference. 
A

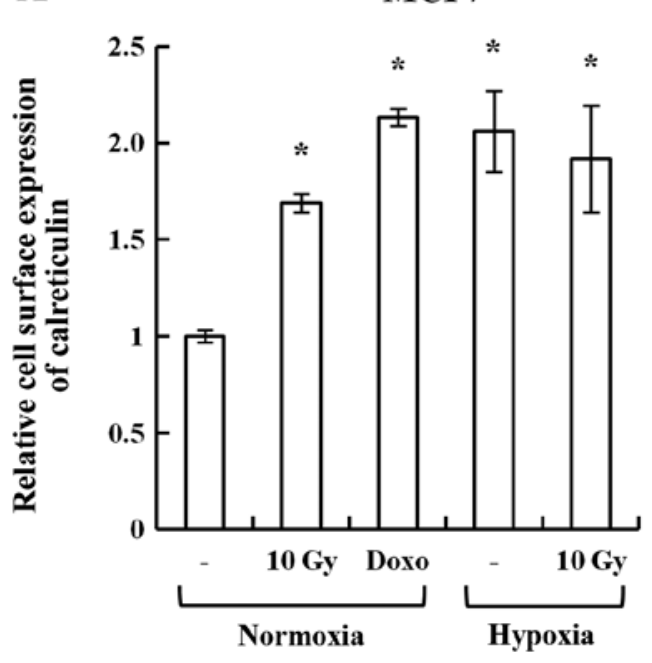

C

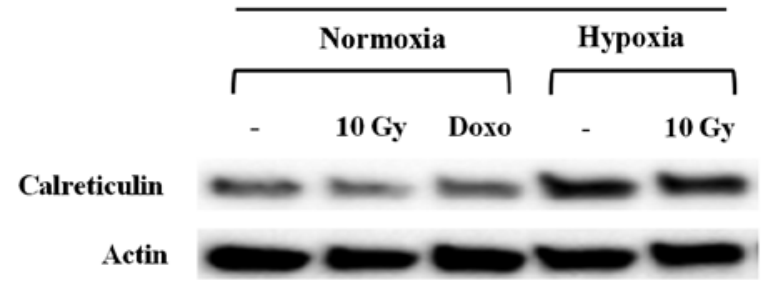

B

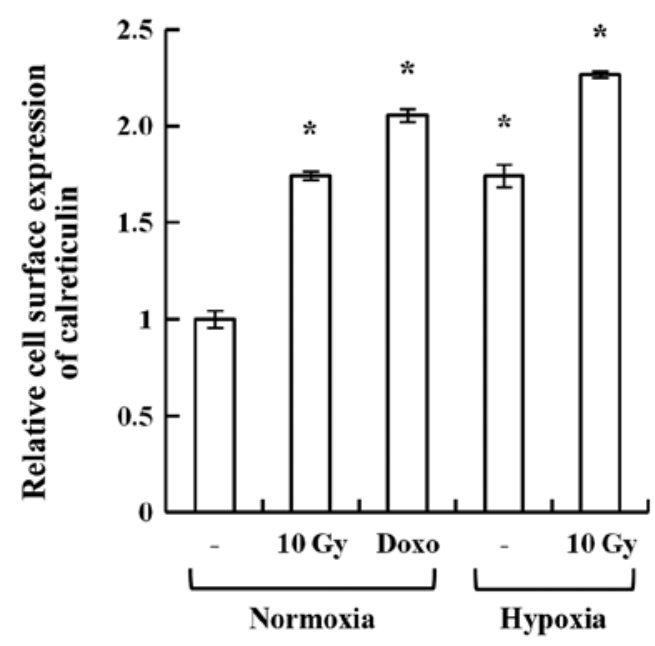

D

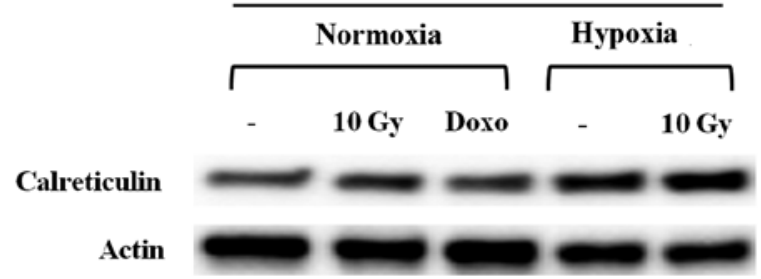

Figure 1. Hypoxia induces the cell surface exposure of calreticulin in human breast cancer cell lines. MCF7 and MDA-MB-231 cells were treated as indicated (IR, 10 Gy irradiation; doxorubicin, $100 \mathrm{nM}$; hypoxia, $1 \% \mathrm{O}_{2}$ ). At $24 \mathrm{~h}$ post-treatment, the cells were analyzed by FACS and western blotting. Cell surface exposure of calreticulin in (A) MCF7 and (B) MDA-MB-231 was analyzed by FACS using an anti-calreticulin antibody. The relative exposure is expressed as the MFI \pm SD. Total cell lysates of (C) MCF7 and (D) MDA-MB-231 were subjected to western blot analysis using an anti-calreticulin antibody. ${ }^{*}<0.05$ vs. normoxia-no treatment group. Doxo, doxorubicin; FACS, fluorescence-activated cell sorting; MFI, median fluorescence intensity.

\section{Results}

Hypoxia increases the cell surface exposure of calreticulin in human and mouse breast cancer cell lines in an ER stress-dependent manner. To investigate whether hypoxia induced the cell surface exposure of calreticulin, the human breast cancer cell lines MCF7 and MDA-MB-231 were cultured under $1 \% \mathrm{O}_{2}$ hypoxic conditions. Irradiation (10 Gy) and doxorubicin, which are known inducers of the cell surface exposure of calreticulin, were used as positive controls $(9,10)$. Hypoxia, irradiation and doxorubicin induced the cell surface exposure of calreticulin in MCF7 and MDA-MB-231 cells (Fig. 1A and B). Western blot analysis suggested that hypoxia not only induced cell surface exposure of calreticulin but also its total expression (Fig. 1C and D).

Furthermore, the present study investigated a mouse breast cancer cell line to determine a similar pattern of results. Using 4TO7, a balb/c-derived breast cancer cell line, it was identified that hypoxia induced both the cell surface exposure and total expression of calreticulin in 4TO7 cells, which was similar to the effects observed in human breast cancer cell lines (Fig. 2A). The cell surface exposure of calreticulin has been reported to be ER stress-dependent (10). Consistent with the previous study, the chemical chaperones TUDCA and 4-PBA decreased hypoxia-induced cell surface exposure of calreticulin (Fig. 2B). These results suggest that hypoxia induced ER stress, which resulted in enhanced cell surface exposure of calreticulin.
Hypoxia-induced cell surface exposure of calreticulin is associated with enhanced immunogenicity of the 4 TO7 mouse breast cancer cell line. To investigate whether the hypoxia-induced cell surface exposure of calreticulin enhanced anticancer immunogenicity, a mouse experiment was performed. The results demonstrated that $\sim 60 \%$ of $4 \mathrm{TO} 7$ cells died after 2 days of culture under $1 \% \mathrm{O}_{2}$ hypoxic conditions, based on annexin $\mathrm{V}$ and propidium iodide staining analysis (data not shown). The left thighs of 6-week-old mice were subcutaneously injected (vaccinated) with $1 \times 10^{6}$ dying 4 TO7 cells. At 7 days post-injection, the right thighs were subcutaneously injected (challenged) with $5 \times 10^{5}$ live $4 \mathrm{TO} 7$ cells to induce tumor growth. Tumor growth in the right thighs of mice vaccinated with hypoxia-treated 4TO7 cells was inhibited as efficiently as in the groups exposed to irradiation (10 Gy) and doxorubicin. However, tumor growth was not suppressed in mice vaccinated with 4TO7 cells treated with PBS and cisplatin, which is a poor inducer of immunogenic cell death (Fig. 3A). Additionally, analysis of tumor-free mice (mice with tumor sizes $<150 \mathrm{~mm}^{3}$ were considered tumor-free since, empirically, it is very difficult to measure the tumor volume below $150 \mathrm{~mm}^{3}$ ) demonstrated that the tumor growth in the hypoxia group was inhibited as efficiently as in the irradiation (10 Gy) and doxorubicin groups (Fig. 3B). These results suggest that hypoxia may have induced immunogenic cell death by enhancing the cell surface exposure of calreticulin. 
A

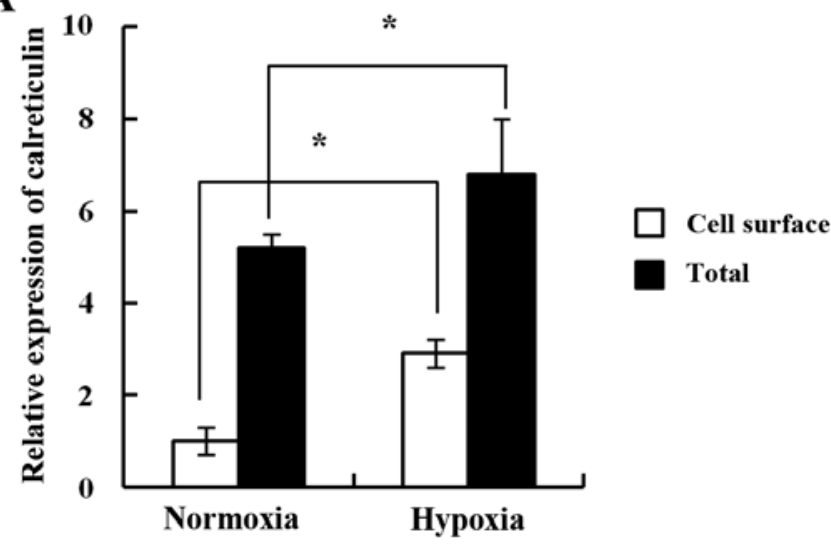

B

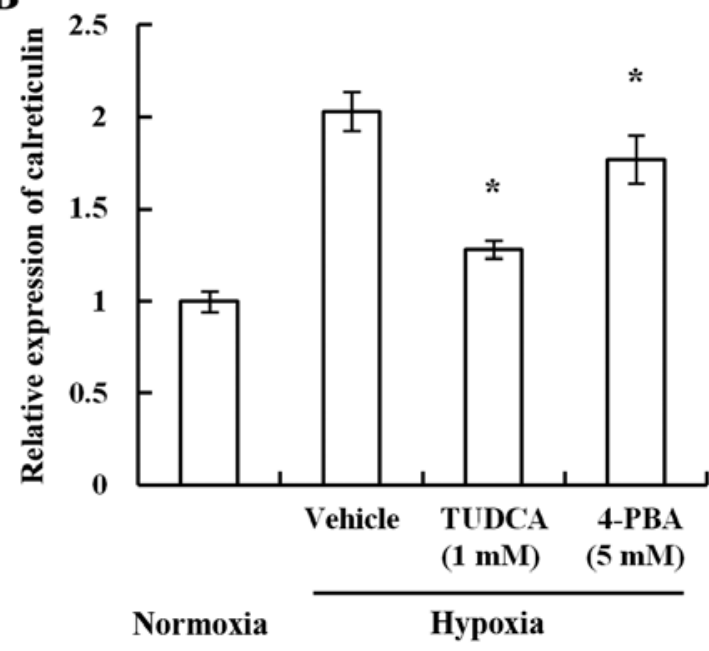

Figure 2. Hypoxia induces the cell surface exposure of calreticulin in a 4TO7 mouse breast cancer cell line in an endoplasmic reticulum stress-dependent manner. (A) 4TO7 cells were treated as indicated (hypoxia, 1\% $\mathrm{O}_{2}$ ). At $24 \mathrm{~h}$ post-treatment, the cells were analyzed by FACS using an anti-calreticulin antibody. For the analysis of cell surface exposure, cells were harvested and stained with anti-calreticulin antibody and fluorescein-labeled anti-rabbit IgG. For total cell expression of calreticulin, the cells were harvested and permeabilized with Cytofix/Cytoperm, followed by staining with anti-calreticulin antibody and fluorescein-labeled anti-rabbit IgG. The relative exposure is shown as the mean MFI \pm SD. Compared with the normoxia groups, both cell surface exposure and total expression of calreticulin in the hypoxia groups were statistically significant. ${ }^{*} \mathrm{P}<0.05$, as indicated. (B) $4 \mathrm{TO} 7$ cells were cultured in hypoxic conditions, with or without chemical chaperones. Cells were analyzed by FACS using an anti-calreticulin antibody. The relative exposure is shown as the mean $\mathrm{MFI} \pm \mathrm{SD} .{ }^{*} \mathrm{P}<0.05$ vs. hypoxia-vehicle group. 4-PBA, 4-phenylbutyrate; FACS, fluorescence-activated cell sorting; IgG, immunoglobulin G; MFI, median fluorescence intensity; TUDCA, tauroursodeoxycholic acid.

Hypoxia increases the cell surface exposure of calreticulin but not CD47, an anti-phagocytic signal. The cell surface exposure of calreticulin is an 'eat me' signal, prompting the recognition and removal of dying tumor cells by phagocytes $(9,14)$. By contrast, the cell surface exposure of CD47 works as a 'do not eat me' signal, which antagonizes the function of calreticulin $(9,14)$. Therefore, the present study examined whether hypoxia modulated the cell surface exposure of CD47 in 4TO7 cells. Irradiation efficiently induced the cell surface exposure of calreticulin and CD47 (4.4-fold for calreticulin and 2.6-fold for CD47 vs. no treatment on day 2; Fig. 4). It is possible that the cell surface exposure of calreticulin induced by irradiation was partially counteracted by the enhanced cell surface exposure of CD47. Hypoxia also induced the cell surface exposure of calreticulin but not that of CD47 (2.8-fold for calreticulin and 1.1-fold for CD47 vs. no treatment on day 2 ; Fig. 4). Therefore, calreticulin surface expression induced by hypoxia may work as an 'eat me' signal effectively, without being antagonized by CD47. Overall, the results suggest that hypoxia may have induced immunogenic cell death by enhancing the cell surface exposure of calreticulin in an ER stress-dependent manner, although hypoxia elicits numerous alterations in the cancer microenvironment, which are unfavorable for the induction of antitumor immunogenicity. Further investigations are required to elucidate the cumulative effect of hypoxia-induced alterations in the cancer microenvironment on the immunogenicity of cancer cells.

\section{Discussion}

Apoptotic cells are removed by phagocytic cells to maintain homeostasis. Phosphatidylserine (PS) on the cell surface of apoptotic cells is a well-known signal recognized by phagocytes $(15,16)$. It is referred to as the 'eat me signal' $(15,16)$.
Gardai et al (14) have demonstrated that calreticulin also acts as an 'eat me signal' for phagocytes. Unlike PS, which is involved in anti-inflammatory and anti-immunogenic responses, the exposure of calreticulin on the apoptotic cell surface induces immunogenic cell death (9). Therefore, anticancer therapies, which induce cell surface exposure of calreticulin during apoptosis, lead to immunogenic cancer cell death. However, calreticulin is also present on the cell surface of live cells, which are not taken up by phagocytes, suggesting a role of specific regulatory mechanisms in the process. CD47 has been demonstrated to act as a 'do not eat me' signal (14). It prevents the uptake of calreticulin-expressing live cells by phagocytes (14). Therefore, CD47 is used as an anti-phagocytic signal in the immune evasion of cancer cells. An anti-CD47 antibody has been developed to enhance anticancer immunity by modulating the balance between pro- and anti-phagocytic signals (17).

Calreticulin is a highly-conserved $46 \mathrm{kDa}$ protein predominantly located in the ER due to the presence of the ER retrieval signal (KDEL) at the C-terminal (18). Calreticulin is a multifunctional protein with $\mathrm{Ca}^{2+}$-binding and chaperone activities important for numerous biological processes, including $\mathrm{Ca}^{2+}$ homeostasis, cellular signaling and protein folding (19-21). Since the $\mathrm{Ca}^{2+}$ signaling pathway is important for T-cell receptor activation, calreticulin contributes to the modulation of the $\mathrm{T}$ cell-mediated adaptive immune response (22). Therefore, calreticulin induces immune responses via extracellular and intracellular signals. In addition to its role in immunogenic cell death in anticancer therapies, calreticulin has been revealed to be involved in a number of aspects of cancer biology, including cancer cell proliferation, differentiation of neuroblastoma and cancer cell migration (23).

Hypoxia is an important obstacle to anticancer therapies since it induces a number of metabolic alterations associated 
A

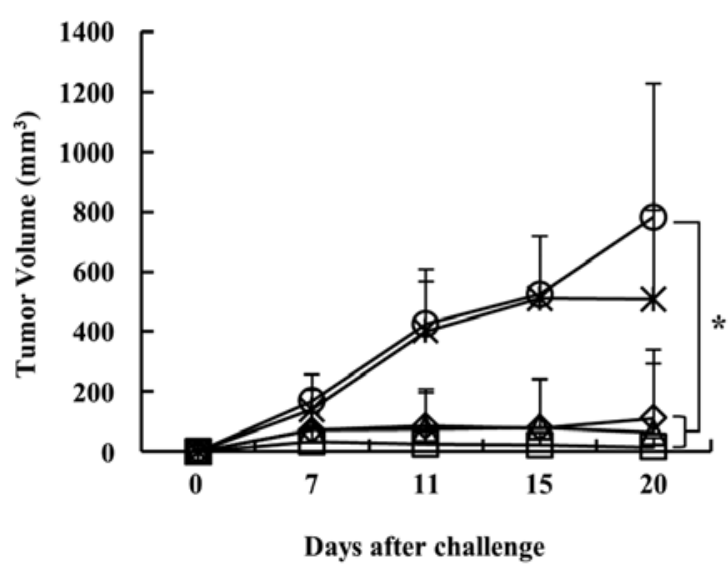

B

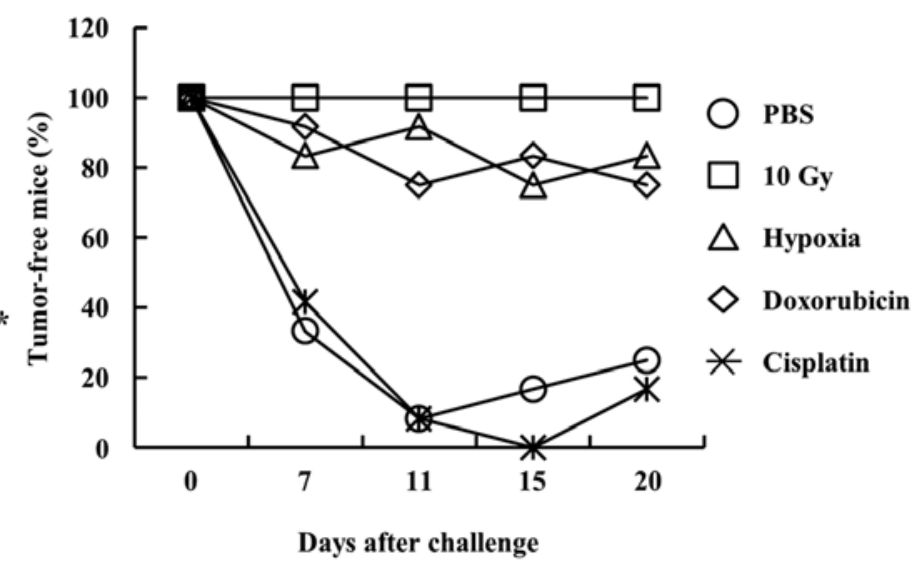

Figure 3. 4TO7 cells dying from hypoxia are immunogenic in mice. A total of $1 \times 10^{6} 4 \mathrm{TO} 7$ cells treated for 2 days with the indicated methods (irradiation, $10 \mathrm{~Gy}$; doxorubicin, $2 \mu \mathrm{M}$; cisplatin, $300 \mu \mathrm{M}$; hypoxia, $1 \% \mathrm{O}_{2}$ ) were subcutaneously injected (vaccinated) in the left thighs of mice. At 7 days post-injection, $5 \times 10^{5}$ live $4 \mathrm{TO} 7$ cells were injected (challenged) into the right thighs. (A) Tumor growth in the right thighs. Tumor volume is expressed as the mean \pm SD. Compared with the PBS group, the doxorubicin, 10 Gy and hypoxia groups were statistically significantly different. (B) Percentage of right thigh tumor-free mice per group. Mice with tumor sizes $<150 \mathrm{~mm}^{3}$ were considered to be tumor-free. ${ }^{*} \mathrm{P}<0.05$.

A

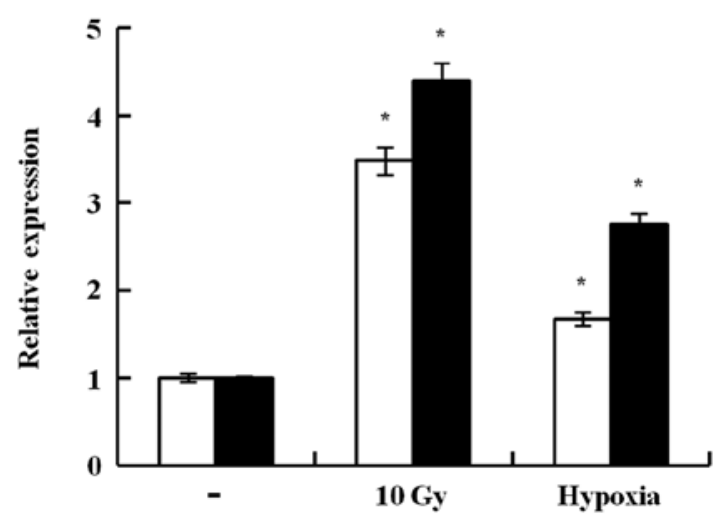

B

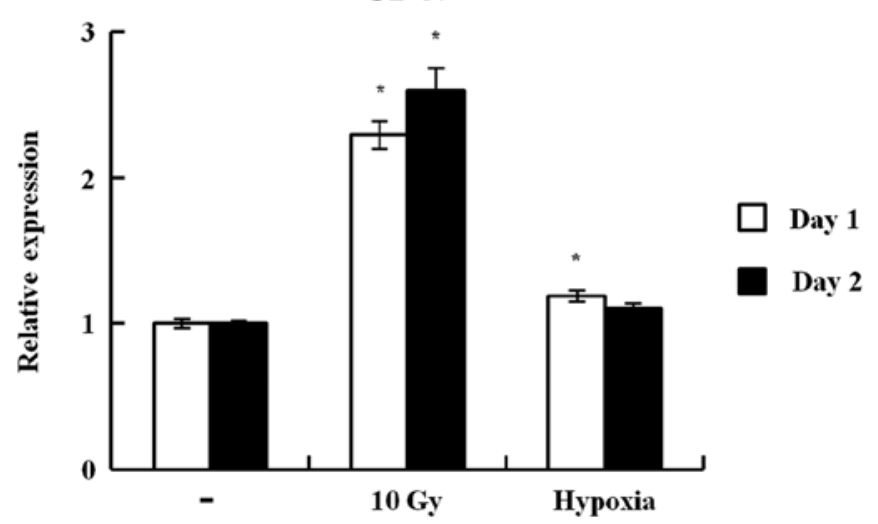

Figure 4. Hypoxia induces the cell surface exposure of calreticulin but not CD47, an anti-phagocytic signal. 4TO7 cells were treated as indicated (irradiation, $10 \mathrm{~Gy}$; hypoxia, $1 \% \mathrm{O}_{2}$ ). At the indicated time points, the cells were analyzed by fluorescence-activated cell sorting using (A) anti-calreticulin or (B) CD47 antibodies. The relative exposure was expressed as the mean median fluorescence intensity $\pm \mathrm{SD}$. ${ }^{*} \mathrm{P}<0.05$ vs. control (no treatment on day 1 or 2 ).

with resistance to apoptosis in cancer cells. Alterations in cancer cells induced by hypoxia have also been associated with immune evasion mechanisms (5-8). Interleukin 10, transforming growth factor- $\beta-\beta$ and vascular endothelial growth factor secreted by cancer cells, and ER stress induced in cancer cells under hypoxic conditions are associated with immune suppression in the tumor microenvironment (5-8). The results of the present study appear to be inconsistent with the results of previous studies (5-8). One may propose that hypoxia-induced alterations in cancer cells result in evasion of immune surveillance and resistance to immune responses. However, other alterations may lead to the immunogenic cell death of cancer cells in a hypoxic microenvironment. Immune evasion or resistance to immune responses may be determined by the cumulative alterations induced by hypoxia. The present study revealed that hypoxia induced immunogenic cell death of cancer cells in an ER stress-dependent manner. This observation is supported by previous studies suggesting that lysates derived from cancer cells cultured at $5 \% \mathrm{O}_{2}$ were improved sources of cancer vaccine antigen than those obtained at $20 \% \mathrm{O}_{2}(24,25)$. Although these studies did not explore the mechanisms underlying the phenomenon, they are similar to the findings of the present study, suggesting that culture conditions at oxygen concentrations $<20 \%$ may enhance the immunogenicity of cancer cells. Future studies may investigate whether the cell surface exposure of calreticulin is induced in cancer cells cultured at 2-5\% oxygen concentrations, which is higher than the oxygen concentration used in the present study. In summary, the results suggest that hypoxia induced favorable and unfavorable alterations in terms of anticancer immunity. Elucidation of the exact mechanisms may facilitate the design of effective anticancer immunotherapies.

\section{Acknowledgements}

The 4 TO7 cells were provided by Dr. Wook Jin at Gachon University (Incheon, Korea). 


\section{Funding}

The present study was supported by the Dongnam Institute of Radiological and Medical Sciences grant funded by the Korean government (MSIT) (grant no. 50491-2015).

\section{Availability of data and materials}

The datasets used and/or analyzed during the current study are available from the corresponding author on reasonable request.

\section{Authors' contributions}

YKH, JSK, WSJ and CGL were involved in the conceptualization of the study and in the methodology. YKH, GYP and MJB performed the experiments. YKH and CGL were involved in data analysis and the writing of the original draft. JSK and WSJ were involved in the writing, reviewing and editing of the manuscript. JSK, WSJ and CGL supervised the study. WSJ and CGL were involved in funding acquisition. All aforementioned authors participated in the conception and design of the study. All authors have read and approved the final manuscript.

\section{Ethics approval and consent to participate}

Animal studies were approved by the Ethics Committee on the Use and Care of Animals of the Dongnam Institute of Radiological and Medical Sciences (Busan, Republic of Korea; approval no. DIRAMS AEC-2015-008).

\section{Patient consent for publication}

Not applicable.

\section{Competing interests}

The authors declare that they have no competing interests.

\section{References}

1. Evans SM and Koch CJ: Prognostic significance of tumor oxygenation in humans. Cancer Lett 195: 1-16, 2003.

2. Le QT, Denko NC and Giaccia AJ: Hypoxic gene expression and metastasis. Cancer Metastasis Rev 23: 293-310, 2004.

3. Majmundar AJ, Wong WJ and Simon MC: Hypoxia-inducible factors and the response to hypoxic stress. Mol Cell 40: 294-309, 2010.

4. Koumenis C and Wouters BG: 'Translating' tumor hypoxia: Unfolded protein response (UPR)-dependent and UPR-independent pathways. Mol Cancer Res 4: 423-436, 2006.

5. Gabrilovich D: Mechanisms and functional significance of tumour-induced dendritic-cell defects. Nat Rev Immunol 4: 941-952, 2004.

6. Hao NB, Lü MH, Fan YH, Cao YL, Zhang ZR and Yang SM: Macrophages in tumor microenvironments and the progression of tumors. Clin Dev Immunol 2012: 948098, 2012.
7. Mahadevan NR, Anufreichik V, Rodvold JJ, Chiu KT, Sepulveda $\mathrm{H}$ and Zanetti M: Cell-extrinsic effects of tumor ER stress imprint myeloid dendritic cells and impair $\mathrm{CD}^{+} \mathrm{T}$ cell priming. PLoS One 7: e51845, 2012.

8. Zanetti M, Rodvold JJ and Mahadevan NR: The evolving paradigm of cell-nonautonomous UPR-based regulation of immunity by cancer cells. Oncogene 35: 269-278, 2016.

9. Obeid M, Tesniere A, Ghiringhelli F, Fimia GM, Apetoh L, Perfettini JL, Castedo M, Mignot G, Panaretakis T, Casares $\mathrm{N}$, et al: Calreticulin exposure dictates the immunogenicity of cancer cell death. Nat Med 13: 54-61, 2007.

10. Panaretakis T, Kepp O, Brockmeier U, Tesniere A, Bjorklund AC, Chapman DC, Durchschlag M, Joza N, Pierron G, van Endert P, et al: Mechanisms of pre-apoptotic calreticulin exposure in immunogenic cell death. EMBO J 28: 578-590, 2009.

11. Lee CG, Park GY, Han YK, Lee JH, Chun SH, Park HY, Lim KH,

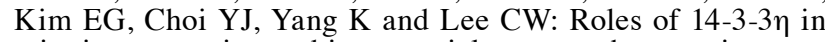
mitotic progression and its potential use as a therapeutic target for cancers. Oncogene 32: 1560-1569, 2013.

12. Park GY, Han JY, Han YK, Kim SD, Kim JS, Jo WS, Chun SH, Jeong DH, Lee CW, Yang K and Lee CG: 14-3-3 eta depletion sensitizes glioblastoma cells to irradiation due to enhanced mitotic cell death. Cancer Gene Ther 21: 158-163, 2014.

13. Aslakson CJ and Miller FR: Selective events in the metastatic process defined by analysis of the sequential dissemination of subpopulations of a mouse mammary tumor. Cancer Res 52: 1399-1405, 1992.

14. Gardai SJ, McPhillips KA, Frasch SC, Janssen WJ, Starefeldt A, Murphy-Ullrich JE, Bratton DL, Oldenborg PA, Michalak M and Henson PM: Cell-surface calreticulin initiates clearance of viable or apoptotic cells through trans-activation of LRP on the phagocyte. Cell 123: 321-334, 2005.

15. Fadok VA, Bratton DL, Guthrie L and Henson PM: Differential effects of apoptotic versus lysed cells on macrophage production of cytokines: Role of proteases. J Immunol 166: 6847-6854, 2001.

16. Hoffmann PR, Kench JA, Vondracek A, Kruk E, Daleke DL, Jordan M, Marrack P, Henson PM and Fadok VA: Interaction between phosphatidylserine and the phosphatidylserine receptor inhibits immune responses in vivo. J Immunol 174: 1393-1404, 2005.

17. Chao MP, Jaiswal S, Weissman-Tsukamoto R, Alizadeh AA, Gentles AJ, Volkmer J, Weiskopf K, Willingham SB, Raveh T, Park CY, et al: Calreticulin is the dominant pro-phagocytic signal on multiple human cancers and is counterbalanced by CD47. Sci Transl Med 2: 63ra94, 2010.

18. Michalak M, Corbett EF, Mesaeli N, Nakamura K and Opas M: Calreticulin: One protein, one gene, many functions. Biochem J 344: 281-292, 1999.

19. Dedhar S: Novel functions for calreticulin: Interaction with integrins and modulation of gene expression? Trends Biochem Sci 19: 269-271, 1994.

20. Leung-Hagesteijn CY, Milankov K, Michalak M, Wilkins J and Dedhar S: Cell attachment to extracellular matrix substrates is inhibited upon downregulation of expression of calreticulin, an intracellular integrin alpha-subunit-binding protein. J Cell Sci 107: 589-600, 1994.

21. Raghavan M, Wijeyesakere SJ, Peters LR and Del Cid N: Calreticulin in the immune system: Ins and outs. Trends Immunol 34: 13-21, 2013.

22. Porcellini S, Traggiai E, Schenk U, Ferrera D, Matteoli M, Lanzavecchia A, Michalak M and Grassi F: Regulation of peripheral $\mathrm{T}$ cell activation by calreticulin. J Exp Med 203: 461-471, 2006.

23. Lu YC, Weng WC and Lee H: Functional roles of calreticulin in cancer biology. Biomed Res Int 2015: 526524, 2015.

24. Olin MR, Andersen BM, Litterman AJ, Grogan PT, Sarver AL, Robertson PT, Liang X, Chen W, Parney IF, Hunt MA, et al: Oxygen is a master regulator of the immunogenicity of primary human glioma cells. Cancer Res 71: 6583-6589, 2011.

25. Olin MR, Andersen BM, Zellmer DM, Grogan PT, Popescu FE, Xiong Z, Forster CL, Seiler C, SantaCruz KS, Chen W, et al: Superior efficacy of tumor cell vaccines grown in physiologic oxygen. Clin Cancer Res 16: 4800-4808, 2010. 